\title{
微孔材料理论模拟的进展与展望
}

\author{
孙䂞双伟侨* \\ (中国科学院大连化学物理研究所 分子反应动力学国家重点实验室 大连 116023)
}

\begin{abstract}
摘要 微孔材料是指孔径小于 $2 \mathrm{~nm}$ 的多孔材料, 被广泛应用于非均相催化、吸附、分离、储气等先进工艺中. 按照组 成结构分类，由于这些材料在元素组成及结构特征上的多样性，因此原则上能够合成的此类材料数目巨大. 仅通过实 验手段无法有效地对这些具有潜在应用价值的材料进行研究. 随着计算机资源与数值计算方法的迅速发展, 理论计算 方法研究微孔材料不仅可以提供分子水平上对材料特性的认知, 而且可以从微观尺度上揭示实验机理, 有利于建立结 构与性能的对应关系, 从而推动新型微孔材料的设计与开发. 综述了近年来针对各种类型微孔材料的理论研究方法及 最新的理论研究成果. 指出了理论方法在微孔材料研究进程中存在的主要问题、发展前景及今后的研究方向.
\end{abstract}

关键词 微孔材料; 理论模拟; 氢气吸附; 甲烷吸附; 二氧化碳吸附

\section{Progress and Prospect of Theoretical Simulation of Microporous Materials}

\author{
Sun, Lei Deng, Weiqiao* \\ (Dalian Institute of Chemical Physics, Chinese Academy of Sciences, Dalian 116023, China)
}

\begin{abstract}
Microporous materials, which refers to the porous materials with pores of less than $2 \mathrm{~nm}$, have been widely used for heterogeneous catalysis, adsorption, separation, gas storage and other numbers of advanced applications. Their high-profile application is mainly focused in the field of energy and environment research, such as the storage and separation of hydrogen, carbon dioxide and methane. According to the compositions and structures, common microporous materials include molecular sieves, porous carbon materials, metal-organic framework compounds (MOF) and microporous organic polymer (MOP). Due to the diversity of element components and structure characteristics, the number of the microporous materials, which can be synthesized in principle, is considerably large. It is impossible to study these materials only by means of experimental methods. With the rapid development of computing power and numerical methods, the theoretical methods used in the studies of microporous materials not only provide the material properties at the molecular level, but also reveal the micro-scale experimental mechanism. Therefore, it is beneficial for establishing the corresponding relationship between the material structures and their properties, leading to promoting the design and development of novel microporous materials. Currently, the accurate theoretical simulations firstly calculate the intermolecular interactions between the key moiety originated from the microporous material and the target molecule through the computational method of quantum chemistry, thereby acquired the potential energy curve of the system. Then the van der Waals interaction parameters of the force field were fitted. Based on the force field, the processes of gas adsorption in the porous materials were simulated by Grand-Canonical Monte-Carlo (GCMC) method. Good agreements between GCMC simulation results and experimental data for adsorption isotherms and heats of adsorption have been observed in many studies. This paper reviews the theoretical methods recently used in the study of the various microporous materials and the latest theoretical research findings. Moreover, the main problems, development prospects and the direction for future research in the study of microporous materials are pointed out.
\end{abstract}

Keywords microporous materials; theoretical simulation; hydrogen adsorption; methane adsorption; carbon dioxide capture

\section{1 引言}

多孔材料是一种由相互贯通或封闭的孔洞构成网 络结构的材料. 按照孔径大小, 多孔材料可以分为微孔 (孔径小于 $2 \mathrm{~nm}$ ) 材料、介孔(孔径 2 $50 \mathrm{~nm}$ ) 材料和大孔 (孔径大于 $50 \mathrm{~nm}$ ) 材料, 其中微孔材料是当前理论模拟 研究的重点与前沿. 比较常见的微孔材料有分子篎、多
孔碳材料、金属有机框架化合物(MOF) ${ }^{[1]}$ 和微孔高分子 $(\mathrm{MOP})^{[2]}$.

分子篮为含有微孔结构的硅铝氧化物晶体, 通用的 分子式为 $\mathrm{M}_{x} /{ }^{n+}\left[\left(\mathrm{AlO}_{2}\right)_{x}\left(\mathrm{SiO}_{2}\right)_{y}\right]^{x-}$. 其结构特点为正四面 体 $\mathrm{SiO}_{4}$ 与 $\mathrm{AlO}_{4}$ 结构单元通过氧原子链接组合成高度规 整的孔径在 0.3 到 $1.2 \mathrm{~nm}$ 的孔道结构. 图 1 为典型的分 子篮结构(框架类型代码为 $\mathrm{MFI}$ ). 因为分子篮由 $\mathrm{Si}-\mathrm{O}$

\footnotetext{
* E-mail: dengwq@dicp.ac.cn; Tel.: 0411-84379571

Received March 19, 2015; published May 22, 2015.

Project supported by the National Natural Science Foundation of China (Nos. 21373202, 21173209).

项目受国家自然科学基金(Nos. 21373202, 21173209)资助.
} 
键与 $\mathrm{Al}-\mathrm{O}$ 键以化学键形式组成, 所以分子篮化学性质 与热性质都比较稳定. 目前分子笁被广泛地应用在分 离、离子交换和催化等领域. 多孔碳材料包括活性碳以 及较新颖的碳纳米管和石墨烯材料. MOF 材料在 2003 年问世，已经成为新的微孔材料成员. MOF 由金属团簇 与有机分子链接而成, 由于其链接方式的多样性使得 MOF 的结构多种多样, 具有大规模应用于储存、分离、 催化与生物领域的前景. 微孔高分子即为具有微孔结构 的聚合物高分子，按特征可分为常规交联高分子 $(\mathrm{HCP})$ 、固有微孔高分子(PIM)、共价有机框架化合物 (COF) 和共轭微孔高分子 (CMP). 其中除 HCP 为较早出 现的高分子材料外, PIM、COF 和 CMP 都是近十年内出 现的新兴微孔材料. 微孔高分子材料主要应用于气体吸 附、分离、催化、电子器件等领域. 表 1 列举了几种微 孔材料的形貌性质及其主要应用.
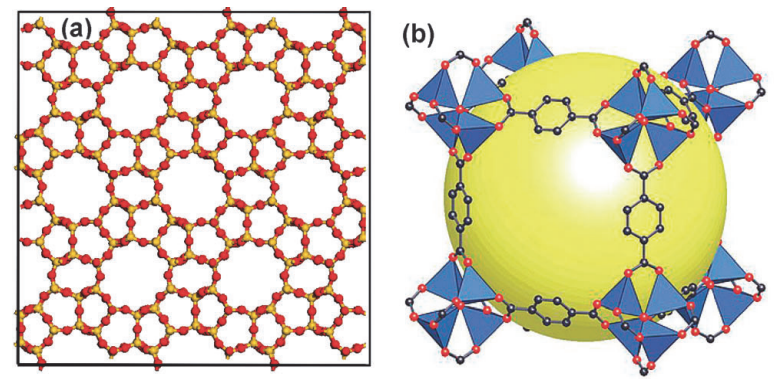

图 1 (a)典型分子篮结构和(b)典型 MOF 结构

Figure 1 (a) Typical structure of zeolite and (b) typical structure of MOF

目前，人们对于微孔材料吸附各种气体分子的机理 仍然不是很清楚, 因此气体在微孔材料中的吸附位点和 吸附机理的研究具有非常重要的意义. 而只通过实验手 段获得相关信息量的限制, 对于气体在微孔材料中复杂 吸附行为的研究存在较大的困难. 另一方面, 由于能够 合成此类材料的数目巨大，仅通过实验方法为特定的材 料选择合适的应用是目前该领域内最大的挑战. 因此, 在微观上对微孔材料清晰的认识极其重要. 而理论模拟 技术手段为我们提供了一种相对成本低、效率高的手段. 随着近年来计算机资源与数值计算方法的迅速发展, 计 算模拟已成为材料科学的重要工具. 在分子层次上的模 拟不仅可以提供实验上无法得到的微观上的深刻理解,
而且可以解释实验机理, 建立结构与性能的对应关系, 从而实现以理论模拟为手段，设计新的材料并指导实 验. 但由于微孔材料结构与组成的复杂性, 微孔材料的 研究工作是目前理论化学研究中的重点和前沿问题. 如 何通过理论方法对氢气、甲烷、二氧化碳等气体分子在 各式微孔材料内的赋存状态、吸附与脱附过程及传输过 程进行研究还需要大量的工作. 本文重点综述近年来相 关理论方法的发展及微孔材料在储氢、甲烷吸附、二氧 化碳捕获与转化等应用中的理论研究进展.

\section{2 理论方法的发展}

为了准确可靠的模拟微孔材料, 首先要获得最基础 的信息一分子间作用力. 气体分子在微纳孔隙内的吸附 过程本质上是气体分子与孔材料分子间及气体分子与 气体分子间协调的相互作用. 目前研究微纳孔隙内分子 间相互作用有两种可靠的方法，一类是第一性原理方 法，另一类是力场方法. 通过第一性原理计算，可以在 微观电子水平上对微孔材料性质及其内部气体相互作 用的本质进行研究. 以往第一性原理方法经常受限于计 算机运算能力的限制无法满足大尺度与高精度的计算 需求，限制了第一性原理方法在该领域的应用. 但随着 计算机运算能力的不断提高, 现在基于第一性原理的动 力学模拟已经逐渐得到广泛的关注, 并已成为当前动力 学模拟的一个新方向. 在研究微孔材料的大部分工作 中, 力场方法仍是这些理论模拟的主要手段. 通常, 力 场参数可由实验结果拟合而得, 然而采用这样的力场研 究具体问题, 会产生较大的偏差. 因此, 目前在较为准 确的模拟工作中, 首先采用量子化学理论方法计算微孔 材料的关键片段与目标分子的相互作用力，获得相应体 系的势能曲线, 并以此拟合力场参数的范德华作用项. 然后，在拟合的力场基础上，通过 Grand Canonical Monte Carlo (GCMC)方法模拟多孔材料中的气体吸附 过程. Deng 等 ${ }^{[3]}$ 最早提出了这一研究方法, 并成功应用 在多孔碳材料的气体吸附理论研究中.

在研究体系间的范德华作用力时，一般采用较高精 度的量子化学计算方法, 比如 MP2, CCSD等. 早期的密 度泛函理论方法如 B3LYP、PW 等对范德华作用力描述

表 1 多孔材料性质与应用

Table 1 Properties and applications of porous materials

\begin{tabular}{|c|c|c|c|c|}
\hline & Pore size/nm & Surface area $/\left(\mathrm{m}^{2} \cdot \mathrm{g}^{-1}\right)$ & Building blocks & Major applications \\
\hline Zeolites & $0.3 \sim 1.2$ & $200 \sim 500$ & $\mathrm{SiO}_{4}, \mathrm{AlO}_{4}$ & Separation, catalysis, ion exchange, water treatment \\
\hline Carbon & $0.3 \sim 50$ & 3000 & C6 rings & Separation and storage, catalysis support, water treatment \\
\hline MOFs & $0.3 \sim 4.5$ & 6500 & $\begin{array}{l}\text { Metal clusters, } \\
\text { organic linkers }\end{array}$ & $\begin{array}{c}\text { Separation and storage, catalysis, ion exchange, sensing, } \\
\text { imaging, drug delivery }\end{array}$ \\
\hline COFs & $0.3 \sim 4.5$ & 4000 & Organic linker & Separation and storage \\
\hline CMPs & $0.4 \sim 1.5$ & 6500 & Conjugated bonds & Separation and storage, catalysis, electronics \\
\hline
\end{tabular}


不准确. 为了研究较大体系的范德华作用力, 研究人员 相继发展出能够准确描述范德华作用力的密度泛函理 论方法, 比如 X3LYP, M06 等. 其中 Xu 等 ${ }^{[4]}$ 发展的 X3LYP 方法是在这一领域中较早的代表性工作.

在获得精确的力场参数后, 材料性能可以通过 Monte Carlo (MC)方法或分子动力学(MD)方法进行模 拟. $\mathrm{MC}$ 方法是一种产生代表性分子或原子排列的随机 方法, 可以非常有效地模拟微孔中气体吸附过程的热力 学性质. 常用到的 GCMC 模拟方法在固定的体积、温度 和化学势情况下, 允许吸附分子在吸附态与非吸附态间 进行交换, 从而能快速获得分子的吸附等温曲线. 通过 GCMC 模拟, 可以获得分子在微孔材料中的吸附量、 Henry 常数、吸附焓等相关热力学性质. MD 方法则是根 据牛顿第二定律来模仿分子在力场作用下的运动. 在给 定的温度下，初始的分子运动速率根据 Maxwell-Boltzmann 分布确定. 在每次循环时, 分子间 作用力由力场参数计算得到并求解相应的运动方程, 得 到的速度与位置更新到下次循环中. MD 方法主要应用 于分子在微孔材料中的动力学性质如输运、扩散等方面 的研究中.

值得注意的是, 在模拟微孔材料时, 为了节省计算 资源, 微孔材料的结构一般采用周期性的晶体结构加以 描述.

\section{3 主要应用}

\section{1 氢气吸附}

早在 1970 年, 美国通用汽车公司的技术研究中心 就提出了 “氢经济” 概念. 1976 年美国斯坦福研究院就 开展了 “氢经济” 的可行性研究. 20 世纪 90 年代中期以 来多种因素的汇合增加了氢能经济的吸引力. 这些因素 包括: 持久的城市空气污染、对较低或零废气排放的交 通工具的需求、减少对外国石油进口的需要、二氧化碳 排放和全球气候变化、储存可再生电能供应的需求等. 氢能作为一种清洁、高效、安全、可持续的新能源, 被 视为 21 世纪最具发展潜力的清洁能源, 是人类的战略 能源发展方向.

储氢问题是能够将氢能应用于运输行业的最主要 的急需解决的关键问题, 它代表着材料界最主要的挑战 性问题之一. 现有的储氢方法包括高压气体, 液氢, 复 合氢化物, 金属氢化物, 多孔材料. 但是没有一种方法 能够满足美国能源部对于储氢材料所需要达到的要求. 美国能源部在 2009 年重设了储氢材料的 2015 年目标: 要求系统能够储存 $5.5 \mathrm{wt} \%$ 氢气, 储存密度为 $40 \mathrm{~g} / \mathrm{L}$. 而 储氢材料的储氢量必须超过这一系统目标.

到目前为止, 有不少工作利用计算模拟来发现最佳 微孔储氢材料的设计规则. Frost 等 ${ }^{[5]}$ 预测了 10 种 MOFs 在 120 bar 与 $77 \mathrm{~K}$ 条件下氢气的吸附能力. 模拟发现, 氢气吸附存在 3 个压强区域: 低压条件下, 氢气吸附量
与吸附焓成正比; 中压条件下，吸附量与表面积成正比; 高压条件下，吸附量与自由体积成正比. 随后 Frost 和 Snurr ${ }^{[6]}$ 进一步考察了 $298 \mathrm{~K}$ 条件下氢气在 MOF 材料中 的吸附能力. 他们发现吸附量在所有压力下只与自由体 积成正比. 在对 MOF 材料储氢性能的模拟中, 他们人 为调整了力场中氢气与 $\mathrm{MOF}$ 的 Lennard-Jones 势参数, 研究需要多大的吸附焓能够使材料能在 $298 \mathrm{~K}$ 与 120 bar 的条件下满足储氢目标. 如图 2 所示, 如果新材料的 自由体积在 $1.6 \sim 2.4 \mathrm{~cm}^{3} / \mathrm{g}$ 之间, 那么当其吸附焓在 $10 \sim 15 \mathrm{~kJ} / \mathrm{mol}$ 时, 该材料能够储存 $6 \mathrm{wt} \%$ 的氢气. 该结 论对设计新型微孔材料具有一定的意义.

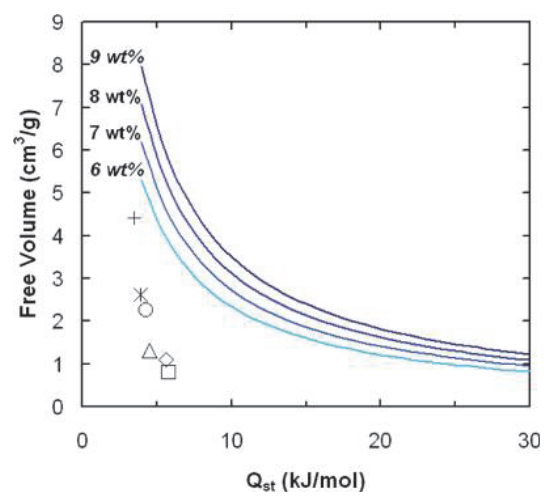

图 2 在 $120 \mathrm{bar}$ 和 $298 \mathrm{~K}$ 条件下储氢目标

Figure 2 Hydrogen storage targets under 120 bar and $298 \mathrm{~K}$

根据研究发现，由于更紧凑的孔径和更多的角落点 有助于提升氢气分子与骨架的相互作用, 一种由两种 MOF 自组装到一起的链状 MOFs 可以提高氢气的吸附 焓. Ryan 等 ${ }^{[7]}$ 利用 GCMC 模拟研究对比了链状 MOFs 与 普通 MOFs 在 120 bar 压强下对氢气的吸附性能. 结果 表明在 $77 \mathrm{~K}$ 条件下, 链状结构可以提高 MOFs 材料对氢 气的吸附能力. 然而, 在室温的条件下, 链状 MOFs 对 氢气的吸附能力远远小于普通的 MOFs. 可见, 这种设 计方法获得的新型 MOF 储氢材料潜力较小.

Han 等 $^{[8]}$ 用 GCMC 方法模拟了 10 个咪唑类沸石材 料(ZIFs，一种典型的 MOF) 在 $77 \mathrm{~K}$ 到 $300 \mathrm{~K}$ 和 $100 \mathrm{bar}$ 条件下对氢气的吸附能力, 并研究了不同的有机功能团 对氢气吸附的影响. 模拟发现在低压的情况下有机官能 团有助于氢气的吸附, 这主要是由于官能团可以提升 MOF 框架与氢气的相互作用. 然而, 官能团的存在会导 致表面积的降低, 在高压下, 这些有机官能团降低了氢 气的吸附.

Bhatia 和 Myers ${ }^{[9]}$ 用 Langmuir 吸附模型评估符合储 氢目标要求的储氢材料最佳吸附焓. 目前的储氢目标要 求在 100 bar 压强下实现氢气的储存, 而在 2 bar 压强下 实现氢气的释放. 考虑到一个完整的储氢和放氢循环过 程, 在 $298 \mathrm{~K}$ 时最优的吸附焓是 $15.1 \mathrm{~kJ} / \mathrm{mol}$. 考虑到一 般微孔材料的吸附焓均小于 $9 \mathrm{~kJ} / \mathrm{mol}$, 所以改变微孔材 料的孔体积、表面积等常规方法无法使微孔材料能够满 足储氢目标. 因此，如何提升目前微孔材料的氢气吸附 
焓是当务之急.

2004 年, Deng 等 ${ }^{[3]}$ 提出利用锂掺杂来提升材料体系 的吸附焓. 他们应用 X3LYP 密度泛函方法研究了 $\mathrm{Li}_{2} \mathrm{C}_{32}$ 团簇对氢气分子的吸附性能, 并在此基础上拟合出能准 确描述较强相互作用的含 Morse 势系数的力场. 量化计 算结果表明, 在 $\mathrm{Li}_{2} \mathrm{C}_{32}$ 团簇中, 锂原子与碳环会产生电 荷分离生成带有部分正电的锂离子, 此时锂离子与氢分 子之间存在较强的相互作用, 其结合能为 $16.7 \mathrm{~kJ} / \mathrm{mol}$. 基于拟合获得的力场, 他们采用 GCMC 方法模拟了设 计出的锂掺杂石墨烯/碳纳米管模型在 $298 \mathrm{~K}$ 条件下对 氢气的吸附. 结果表明, 当设计的材料中石墨烯层间距 在 $10 \AA$ 时, 氢气在 $298 \mathrm{~K}$ 和 $20 \mathrm{bar}$ 的条件下吸附量达到 $6.5 \mathrm{wt} \%$, 从而满足了储氢目标, 如图 3 所示. Han 等 ${ }^{[10]}$ 将这一概念应用到 MOFs 上, 并应用 GCMC 模拟了锂掺 杂 MOFs 的储氢性能. 模拟结果表明当 MOF 中有机链 接基团 $\left(\mathrm{C}_{28} \mathrm{H}_{12}(\mathrm{COO}-)_{2}\right)$ 掺杂 6 个锂时, 体系可以在 100 bar 和 $243 \mathrm{~K}$ 条件下实现 $6 \mathrm{wt} \%$ 的储氢量. 通过掺杂锂提 升体系的吸附焓这一概念被应用到更多的微孔材料设 计中, 比如锂掺杂富勒烯 ${ }^{[1]}$ 、碳纳米结构、多孔石墨烯、 $\mathrm{COF}^{[12]} 、 \mathrm{CMP}^{[13]}$ 等. 同时其他碱金属、碱土金属及氟离 子的掺杂也引起了人们的兴趣. 同时, 过渡态金属掺 杂 ${ }^{[14]}$ 也开始进入到人们的视野. 然而理论发现过渡态 金属原子容易发生聚集, 而碱金属及碱土金属与材料的 作用力要强于其本身的自聚能力, 因此更容易实现原子 程度的分散.
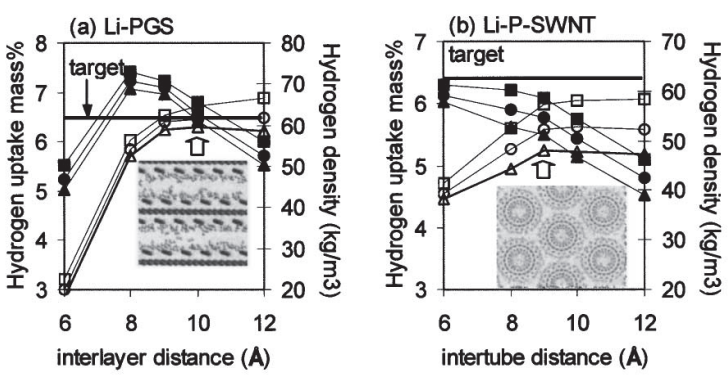

图 3 锂掺杂石墨烯与锂掺杂碳纳米管的储氢性能

Figure 3 Hydrogen storage performances in lithium-doped graphene and carbon nanotubes

2010 年, $\mathrm{Li}$ 等 ${ }^{[13]}$ 合成出了锂掺杂 CMP 材料并测试 了其储氢性能, 其在 $77 \mathrm{~K}$ 和 $1 \mathrm{bar}$ 的情况下储氢量达到 $6.1 \mathrm{wt} \%$, 而未掺杂的 CMP 在同等条件下储氢量仅为 $1.6 \mathrm{wt} \%$. 实验结果证实了锂掺杂可以显著地提升储氢 材料的吸附焓. 2012 年, Konstas 等 $^{[15]}$ 合成了锂掺杂 Porous Aromatic Frameworks (PAF, 一种类似 CMP 结构 的微孔高分子), 测试表明通过锂掺杂, 氢气吸附量增 加了 $22 \%$. 虽然这些实验结果证实了锂掺杂可以显著地 提升微孔材料对氢气的吸附焓, 但是理论计算发现掺杂 锂与氢气的相互作用力为 $16.7 \mathrm{~kJ} / \mathrm{mol}$, 意味着在常温情 况下, 锂掺杂微孔材料同样具有较大的氢气吸附焓. 然 而实验表明, 这些锂掺杂材料室温下的氢吸附小于 0.5 $w t \%$. 锂掺杂体系有待理论与实验工作结合起来的更深 入理解.

相对于掺杂金属锂原子，通过金属醇盐的办法在微 孔材料里引入锂离子与镁离子被认为在实验上更容易 实现. 然而, 计算机模拟锂醇盐功能化 MOFs 的结果却 显示出相互矛盾的结果. Klontzas 等 ${ }^{[16]}$ 认为在 $300 \mathrm{~K}$ 条 件下通过锂醇盐的功能化改造可以显著提升微孔材料 的储氢量, 而 Getman 等 ${ }^{[17]}$ 认为其在 $243 \mathrm{~K}$ 情况下这种 掺杂并不影响储氢量. Getman 等的模拟结果表明锂醇盐 与氢气分子的结合能太低, 仅有 $10 \mathrm{~kJ} / \mathrm{mol}$, 无法有效限 制氢气分子在温和的温度条件下进行的热运动, 达不到 增强氢气吸附的效果. 更进一步研究中, Getman 计算了 镁、锰、镍、铜的金属醇盐与氢气的相互作用，其结合 能分别为 $22,20,78$ 和 $84 \mathrm{~kJ} / \mathrm{mol}$. 这些醇盐功能化的 MOF 材料在氢气吸附性能上表现的各不相同. 其中镍 和铜与第一个氢气分子结合很强, 然而与第二个分子及 以后若干氢气分子的结合却很弱(小于 $10 \mathrm{~kJ} / \mathrm{mol}$ ). 如此, 这两种醇盐功能化的材料在低压时可以吸附较多的氢 分子, 从而不利于氢气的释放过程. 与之相比, 镁醇盐 则具有很大的优势. 计算机模拟结果表明镁醇盐功能化 的 IRMOF-16 材料在 $100 \mathrm{bar}$ 与 $243 \mathrm{~K}$ 条件下可以进行 可逆的氢气吸附, 其储氢量高达 $5.2 \mathrm{wt} \%$. 在实验上, 锂、镁醇盐功能化的 MOFs 材料已经被成功合成, 其对 储氢性能的提升获得了实验结果的验证.

2010 年, Zhou 等 ${ }^{[18]}$ 提出了通过施加外电场提升氢 气吸附焓的方法, 并采用密度泛函理论方法研究了氢分 子与硼氮等材料在施加外电场情况下的相互作用力. 模 拟结果表明, 在 0.045 a.u. 电场的作用下, 氢气分子与嗍 氮材料的相互作用力由 $0.03 \mathrm{eV}$ 提升至 $0.14 \mathrm{eV}$. 这种提 升对高极化性的低维材料比如硼氮片, 硅酸盐片等都有 效.

对于物理吸附储氢材料而言, 不同于甲烷或二氧化 碳的吸附, 努力的重点并不在于追求材料大的表面积与 自由体积, 而在于在保持表面积不变的情况下如何提升 体系对氢气分子的吸附焓. 这是一个仍需要新概念、新 思路的研究领域.

\section{2 甲烷吸附}

尽管天然气(主要成分是甲烷)的燃烧也产生二氧化 碳，但是在整个过程中其产生的二氧化碳量远远低于石 油燃料消耗所产生的. 因此, 甲烷被认为是一种潜在的 石油替代能源. 甲烷储存的目标是在温和温度与 $35 \mathrm{bar}$ 压强条件下, 储存量达到 $35 \mathrm{wt} \%$ (吸附气体重量与总质 量比)或者 $180 \mathrm{~V} / \mathrm{V}$ (吸附气体的体积与微孔材料体积比). 这一目标与压缩天然气的能量密度相当. 不同于储氢材 料, 现有一些微孔材料已经接近或满足甲烷气体的储存 目标. 表 2 列举了几个接近或满足目标的例子. 
表 2 应用于二氧化碳吸附的多孔材料

Table 2 Porous materials applied in $\mathrm{CO}_{2}$ adsorption

\begin{tabular}{|c|c|c|c|c|c|c|c|c|}
\hline & \multirow{2}{*}{$\mathrm{BET} /\left(\mathrm{m}^{2} \bullet \mathrm{g}^{-1}\right)$} & \multirow{2}{*}{ Lang. $/\left(\mathrm{m}^{2} \cdot \mathrm{g}^{-1}\right)$} & \multirow{2}{*}{ Pore volume $/\left(\mathrm{cm}^{3} \cdot \mathrm{g}^{-1}\right)$} & \multirow{2}{*}{ Pressure/bar } & \multirow{2}{*}{ Temp. $/{ }^{\circ} \mathrm{C}$} & \multicolumn{2}{|c|}{ Storage } & \multirow[t]{2}{*}{ Ref. } \\
\hline & & & & & & $\mathrm{wt} \%$ & $V / V$ & \\
\hline ZIF-68 (MOF) & & 1090 & & 1.01 & 298 & 0.7 & 150 & [19] \\
\hline NOTT-107 (MOF) & 1822 & & 0.767 & 35 & 298 & & 185 & [20] \\
\hline COF-102 (COF) & 3620 & 4650 & 1.55 & 35 & 298 & 15.8 & & [21] \\
\hline PPN-4 (CMP) & 6461 & 10063 & 3.04 & 35 & 295 & 21.5 & & [22] \\
\hline
\end{tabular}

Duren 等 ${ }^{[23]}$ 最早使用计算机模拟研究了不同 MOF 材料、分子篮及多孔碳的甲烷吸附量, 吸附焓和表面积. 其结果表明决定甲烷吸附的最重要因素是表面积, 其后 分别是自由体积, 框架密度和吸附焓. 然而, 科研工作 者们更关注哪些材料结构在微观尺度上对甲烷气体的 吸附过程起主要作用. 实验上, 研究人员采用拉曼光谱 对重复网络结构的金属框架化合物(Isoreticular MOFs, IRMOFs)对甲烷的吸附过程进行了研究, 结果表明, 在 IRMOFs 中有机链接基团对甲烷吸附起主要作用. 这一 结论与氢气的吸附恰恰相反, 如前文所述, 在 MOF 材 料对氢气的吸附过程中, 金属团簇对氢气分子的吸附起 主要作用. Babarao 等 ${ }^{[24]}$ 的计算模拟工作也证实了有机 链接基团在甲烷气体吸附过程中起到关键作用.

对于如何提升微孔结构对甲烷气体的吸附能力, 从 理论模拟中提出了若干较新颖的概念. Jhon 等 ${ }^{[25]}$ 报道了 使用计算机模拟研究烷氧基取代的 IRMOF 吸附甲烷的 情况, 他们发现在较低压力的情况下, 烷氧基会导致 IRMOF 的孔体积变小, 从而使甲烷与孔的接触增强, 因 而对甲烷吸附起提升的效果. 然而, 在高压的情况下, 由于总的孔体积减少, 甲烷气体的饱和吸附量会随之减 少. Lan 等 ${ }^{[26]}$ 利用 $\mathrm{QM}$ 与 $\mathrm{GCMC}$ 的模拟方法, 讨论了 COF-102、COF-103 和锂掺杂 COF 材料对甲烷的吸附. 模拟结果表明, 锂掺杂可以将 COF 材料对甲烷的吸附 量提高 2 倍左右. Kowalczyk 等 ${ }^{[27]}$ 提出了对氢气和甲烷 混合气( “Hythane”) 储存的概念, 并利用 GCMC 方法研 究了不同碳材料对氢气与甲烷混合气的储存效果. 研究 结果表明孔径为 $1 \sim 2 \mathrm{~nm}$ 的单壁碳纳米管束对混合气的 储存效果最佳.

另外, 比较有意义的工作是 $\mathrm{Wu}$ 等 ${ }^{[28]}$ 结合实验与 QM 计算, 研究了一系列 MOF $\left(\mathrm{M}_{2}\right.$ (dhtp) (open metal M $=\mathrm{Zn}, \mathrm{Mg}, \mathrm{Mn}, \mathrm{Co}, \mathrm{Ni}$; dhtp=2,5-dihydroxyterephthalate) 中, 未饱和金属位点对甲烷的强力吸附作用, 如图 4 所 示. $\mathrm{M}_{2}(\mathrm{dh}$ tp)框架中富含未饱和金属位点, 这些位点对 甲烷的吸附远远大于经典的 MOF 材料. 更进一步研究 中, $\mathrm{Wu}$ 等 ${ }^{[29]}$ 结合实验与理论方法 $(\mathrm{QM}$ 和 $\mathrm{GCMC}$ 计算模 拟), 研究了 HKUST-1, PCN-11 和 PCN-14等含未饱和金 属位点的 MOF 材料对甲烷气体的吸附能力. 他们发现, 虽然未饱和金属位点对甲烷吸附能力最强, 但是吸附在 金属位点的甲烷数量并不占大多数. 相反, 有机链接基 团对总的甲烷吸附量占主要因素. 该结论说明纳米孔道 与甲烷分子之间的范德华作用力是甲烷气体在微孔材 料中吸附的主要驱动力. 因此, 调整孔道的形状和大小
是提升甲烷吸附的关键，而表面积与修饰基团的活性则 起次要的作用. 不仅如此, $\mathrm{Wu}$ 等 ${ }^{[29]}$ 结合实验, 证实了更 大的有机链接基团将会进一步提升甲烷气体的吸附量. 该研究工作对设计新型 MOF 材料应用于甲烷吸附具有 重要的指导意义.

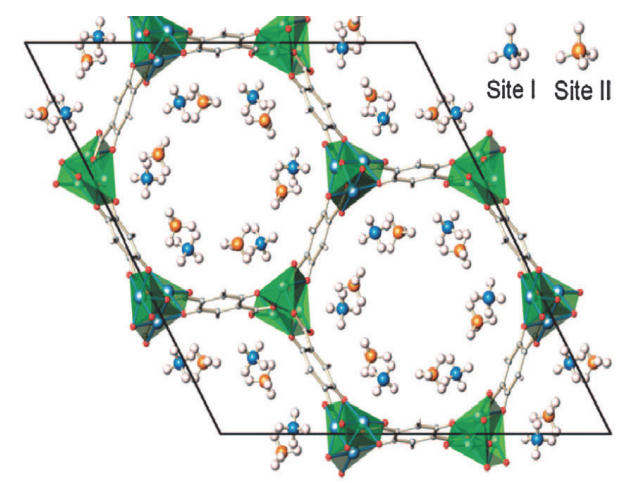

图 4 由实验与理论结合确定的甲烷在 MOF 里吸附位

Figure 4 Adsorption sites of methane in MOF obtained from experimental and theoretical studies

Mendoza-Cortes 等 ${ }^{[30]}$ 结合实验, 应用 $\mathrm{QM}$ 与 $\mathrm{GCMC}$ 的方法模拟了不同 COF 材料对甲烷气体的吸附. 其中, COF-5 与 COF- 8 的理论模拟结果与实验符合很好. 采用 同样的力场参数, 他们设计并预测了多种还未合成的 COF 材料, 并提出 COF-1 是一个潜在的甲烷储存材料, 在 $295 \mathrm{~K}$ 和 $30 \mathrm{bar}$ 的条件下其对甲烷气体的吸附量可以 达到 $195 V / V$, 满足了甲烷储存目标.

最近一个非常重要的成果是 2012 年 Wilmer 等 ${ }^{[31]}$ 发展一套的方法, 他们采用可行的结构单元通过排列组 合的办法, 构建一个含有 137953 种 MOF 结构的数据库. 对其中每一种虚拟的 MOF 材料, 分别计算其孔径分布, 表面积, 并利用 GCMC 方法模拟了其甲烷的储存性能. 通过这样的计算机虚拟篮选，最后得到了 300 种新的 MOF 结构, 其对甲烷储存性能超过目前已知的任何 MOF 材料. 不仅如此, 通过对比和分析数据结果, Wilmer 等揭示了 MOF 结构单元与甲烷储存性能之间的构 效关系. 为了验证理论模拟结果的准确性, Wilmer 等选 择了一个计算机构建的 MOF 结构, 进行了合成与表征, 最终证实了其预测的储存性能. 该研究工作体现了理论 模拟在实验过程中的意义所在.

Llewellyn 等 ${ }^{[32]}$ 结合实验与分子动力学模拟, 发现 某些 MOF 材料随压力变化对甲烷分子的吸附存在 “呼 吸作用”. 科研工作者也开始关注柔性 MOF 材料在甲 
烷气体吸附中的应用.

\section{3 二氧化碳吸附}

自从工业革命以来, 大气中的二氧化碳的浓度增长 了 $40 \%$, 很大程度来源自化石能源的利用与消耗. 伴随 着二氧化碳浓度的持续增长, 由此带来的全球变暖问题 也日益突出. 目前, 碳捕获与封存问题不仅是个科学问 题, 更是一个关乎国计民生的社会问题. 在碳捕获与封 存过程中, 关键步骤是如何在燃烧前后捕获二氧化碳. 在燃烧进行后捕获二氧化碳需要将二氧化碳从氮气与 其混合气中分出; 在燃烧进行前捕获则需要将二氧化碳 从合成气(一氧化碳和氢气)中分离出. 值得注意的是, 分离二氧化碳与甲烷也是天然气化工中的重要步骤.

对以上混合气中二氧化碳气体的捕获，人们提出了 几种有效的办法, 例如有机胺吸收、低温蒸馏、膜分离 与多孔材料吸附等. 其中有机胺吸附是目前已经商业化 的方法, 然而由于操作繁杂, 能耗大以及设备损坏快等 缺点, 增大了该方法捕获二氧化碳的成本. 采用低温蒸 馏技术所需能耗太大. 膜分离的效率较低, 捕获的二氧 化碳浓度很低, 分离有效性不高. 而经过最近几年来的 大力发展, 多孔材料吸附方法取得了长足的进步, 由于 其能耗低、投资少和可大规模化的优势，有望成为下一 代二氧化碳捕获的工业技术.

在理论模拟中, Jiang 等 ${ }^{[33]}$ 应用实验中已经验证的 力场参数模拟了二氧化碳与氮气混合气在钠离子交换 ZSM-5 分子篎中的吸附过程. 预测结果表明二氧化碳在 钠离子交换 ZSM-5 中的选择性吸附能力优于在未进行 钠离子交换的 ZSM-5 材料中. 这主要是由于二氧化碳 具有较大的四极矩, 与钠离子间能够产生较强的相互作 用, 从而增强吸附能力.

Liu 和 $\mathrm{Smit}^{[34]}$ 通过模拟比较了分子篎与 MOF 材料 捕获二氧化碳的能力, 他们分别对比了 MFI, DDR 和 LTA 三种框架类型分子䇻材料与 7 种 MOF 材料 (Cu-BTC, MIL-47, IRMOF-1, -11, -12, -13 和-14). 模拟 结果表明, 虽然 MOF 材料对气体的吸附量较高, 但它 们对二氧化碳与氮气的选择性吸附能力上与分子篮表 现相当. 可见, 二氧化碳所具有的较大的四极矩是分离 二氧化碳与氮气的关键因素, 而孔道、表面积等对二氧 化碳选择性吸附帮助不大.

Babarao 等 ${ }^{[35]}$ 因此提出了采用离子化 MOF 材料 (soc-MOF, rht-MOF, rho-ZMOF)选择性地吸附二氧化碳. 模拟结果表明二氧化碳能较强地与 MOF 材料中的钠离 子产生相互作用. 如果将 MOF 材料中的电荷设为零, 那么吸附的选择性几乎消失. 值得注意的是, 虽然这三 种 MOFs 的自由体积排序为 rho-MOF $<$ soc-MOF $<$ rht-MOF, 其电荷密度排序为 rho-MOF $>$ soc-MOF $>$ rht-MOF, 而吸附选择性排序却为 rho-MOF $>$ soc-MOF $>$ rht-MOF. 这样的结果说明了电荷密度为吸附选择性的 决定因素, 因此离子化 MOF 材料可能是很好的二氧化
碳捕获材料.

比较有意义的工作是 2012 年 Lin 等 ${ }^{[36]}$ 利用 Monte Carlo 和对称性限制的方法来构建了一个含 590000 种虚 拟结构的分子篮和 Zeolitic imidazolate frameworks (ZIF, 一种典型的 MOF)结构的数据库, 并利用 GCMC 方法模 拟这些结构对二氧化碳/氮气的选择性吸附能力, 篮选 出其中几百种具有显著选择性吸附效果的结构, 采用这 些结构的材料能够节省在捕获与储存二氧化碳中所消 耗的 30\% 40\%的能量. 2013 年, Kim 等 ${ }^{[37]}$ 也开展了类 似的研究工作, 他们构建了 87000 种虚拟分子笁结构, 采用 GCMC 模拟了这些结构对二氧化碳与甲烷混合气 的选择性吸附, 最终篮选出可以有效实现选择性吸附的 分子篮结构.

以上的模拟研究工作主要集中在二氧化碳吸附方 面, Krishna 等 ${ }^{[38]}$ 应用 MD 的方法研究了分子笁膜对二氧 化碳与甲烷的分离作用. 计算机模拟结果预测了分子扩 散和渗透中的选择性, 其中 CHA 和 DDR 笼结构表现出 较高的选择性能力. 2010 年, Haldoupis 等 ${ }^{[39]}$ 提出了一种 计算机模拟方法, 他们通过计算气体分子的扩散系数来 确定能够实现气体动力学分离的 MOF 材料. 其中, 分 子的扩散系数是通过孔结构的关键性形状估算获得. 采 用这种方法, Haldoupis 等确定了 500 种 MOF 材料和 160 种分子篮材料具有比较突出的气体分离能力.

就目前情况而言, 对于二氧化碳的捕获与化学利用 的大量研究，基本上都是将两者分开来考虑. 2013 年 $\mathrm{Xie}$ 等 $^{[40]}$ 基于对钴络合的 CMP (Co-CMP)催化二氧化碳 转化机理的量子化学研究结果, 设计并合成了一种共轭 微孔高分子材料, 首次实现了在常温常压下捕获二氧化 碳, 并且同时催化转化二氧化碳为有价值化学品. 在常 温常压条件下, Co-CMP 可以捕获 $79 \mathrm{mg} / \mathrm{g}$ 二氧化碳, 媲 美于优秀的二氧化碳吸附多孔材料, 同时可以催化二氧 化碳与环氧丙烷生成碳酸丙烯酯的反应，经过 $48 \mathrm{~h}$ 反应 产率达 $81 \%$, 选择性 $100 \%$. 在相同反应条件下 $\left(120{ }^{\circ} \mathrm{C}\right.$, $3.0 \mathrm{MPa})$ 与其他催化剂对比, 其催化活性达到商业催化 剂的 10 倍, TOF 高达 $11600 \mathrm{~h}^{-1}$, 超过了目前对该反应报 道的所有催化剂包括均相催化剂. Co-CMP 催化寿命高 达 $1056 \mathrm{~h}$, 当催化活性下降时, 仅通过简单处理(盐浸 泡)就可再生催化剂. 基于这一研究成果, 他们提出一 个新的概念, 常温常压下捕获与转化二氧化碳: 即设计 并找到一种双功能材料, 在常温常压的条件下, 既能实 现二氧化碳的捕获, 同时又能催化转化二氧化碳为有价 值的化学产品. 由于这一过程是在常温常压的条件下进 行, 不需要使用任何高温或高压设备, 因此装置价格低 廉，可以将目前上亿元的设备投资降低到数百万元. 而 且由于常温常压, 这个过程能耗很小, 避免了二氧化碳 的二次排放. 该研究工作对二氧化碳捕获与转换技术的 应用具有重要意义. 


\section{4 其它气体应用}

除氢气、甲烷及二氧化碳之外, 科研工作者也开展 了微孔材料对其它气体吸附与分离的研究工作, 如一氧 化氮、乙炔、乙烯及氧气等. 然而, 相应的计算机模拟 工作进展缓慢, 这主要由于相关体系还没有获得足够的 关注. 随着气体吸附与分离领域的深化与拓展, 这方面 的理论研究工作将会逐步丰富起来.

\section{4 总结与展望}

在过去十年里, 对微孔材料的理论模拟使得人们在 分子水平上对微孔材料性质的认识得到了飞速的发展, 但是目前在这一领域仍然有一些有待解决的问题.

(1) 普适的精确力场目前还不具备. 对于生物体系 来说, 几个力场比如 Amber、Dreiding 等被认为是比较 好的普适的力场. 然而对微孔材料而言, 大部分工作适 用的是对范德华力描述准确度不够的经验力场. 只有有 限的几个工作是用高精度量化计算拟合出的精确分子 力场, 而且这些力场参数只适合于几种特定的原子形 式. 为了提升计算模拟的精度, 一个适用于微孔材料的 普适型分子力场是必不可少的.

(2) 描述柔性 MOF 体系的力场目前还不具备. 目 前大部分的计算模拟处理体系时是以刚性骨架为主, 虽 然这样做提升了计算效率, 然而不能描述柔性 MOF 在 与气体分子作用时的结构改变. 对分子篮而言, 有一些 柔性的分子力场. 然而对于 $\mathrm{MOF}, \mathrm{COF}, \mathrm{CMF}$ 等, 都缺 乏这方面的研究.

(3) 多尺度模拟的方法还不完善. 目前大部分对微 孔材料的模拟都是用周期性条件, 模拟的尺寸也是在 $\mathrm{nm}$ 量级. 而气体分子等在微孔材料里的热力学及动力 学性质是个宏观性质, 所以实验上得到的结果与理论上 的结果往往没有可比性. 为了能更进一步能够预测与指 导实验, 更大尺度的模拟是急需的. 比如在微米量级的 尺度模拟等. 这样一来, 新方法的提出是必不可少的.

(4) 仍需发展大规模计算模拟笁选的方法. 虽然有 几个工作已发展出一些对微孔分子进行大规模计算机 篮选的方法来设计新的材料, 但是这些工作主要是分子 篮或 MOF. 一些新兴微孔材料比如 COF, PIM, CMP 的 篮选方法有待发展.

微孔材料这个领域在过去十年内得到了迅猛的发 展, 一些新出现的材料比如 MOF、COF、CMP 等迅速 占据了材料学的研究热点与前沿, 这些材料的一些新奇 的性能不断被发现. 展望未来十年, 这些新出现的材料 中有一些极有可能得到大规模应用, 并且影响巨大. 从 理论模拟的发展上看, 因为这些新兴材料的可能结构是 上百万种, 潜在的应用也是多种多样, 所以瞄准所需的 材料性能能够进行大规模筛选的理论方法是此领域今 后十年内的最主要挑战.

\section{作者简介}

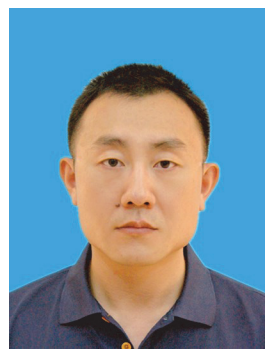

孙磊, 博士, 助理研究员. 2006 年 7 月于吉林大学化学学 院获得学士学位; 同年 9 月进入吉林大学理化所进行研究生 学习, 师从张红星教授; 2011 年 7 月于吉林大学理化所获得博 士学位; 2011 年 8 月至 2013 年 12 月在中国科学院大连化学物 理研究所从事博士后研究. 目前主要研究方向为与能源环境 相关材料的多尺度理论模拟, 包括微孔材料在气体吸附与分 离方面的理论模拟及染料敏化太阳能电池与钙钛矿太阳能电 池器件模拟.

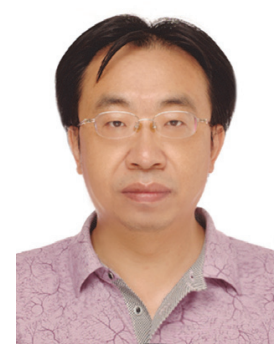

邓伟侨, 博士, 研究员. 1994 年毕业于兰州大学化学系获 学士学位, 1997 年中国科学院大连化学物理研究所获硕士学 位, 2004 年美国加州理工学院获博士学位. 毕业后曾任美国加 州理工学院副研究员、新加坡南洋理工大学助理教授. 2009 年 8 月加入大连化学物理研究所, 入选中国科学院 “百人计划”, 任大连化学物理研究所研究员. 研究工作主要以创新理论方 法为核心，用计算机模拟设计所需性能材料，并有效地合成 所设计的材料. 目前研究方向聚焦在气体捕获微孔材料与太 阳能电池材料.

\section{References}

[1] (a) He, Y. P.; Tan, Y. X.; Zhang, J. Acta Chim. Sinica 2014, 72, 1228 (何燕萍, 谭衍曦, 张健, 化学学报, 2014, 72, 1228.); (b) Jia, J. T.; Wang, L.; Zhao, Q.; Sun, F. X.; Zhu, G. S. Acta Chim. Sinica 2013, 71,1492 . (贾江涛, 王蕾, 赵晴, 孙福兴, 朱广山, 化学学报, 2013, 71, 1492.); (c) Liu, P.; Lian, Y. H.; Li, Z.; Chen, G. J. Acta Chim. Sinica 2014, 72, 942. (刘蓓, 廉源会, 李智, 陈光进, 化学 学报, 2014, 72, 942.); (d) Liu, P.; Tang, L. X.; Lian, Y. H.; Li, Z.; Sun, C. Y.; Chen, G. J. Acta Chim. Sinica 2013, 72, 942. (刘蓓, 唐 李兴, 廉源会, 李智, 孙长宇, 陈光进, 化学学报, 2013, 71, 920.)

[2] (a) Wang, W.; Yan, Z. J.; Yuan, Y.; Sun, F. X.; Zhao, M.; Ren, H.; Zhu, G. S. Acta Chim. Sinica 2013, 71, 1598. (王维, 间卓君, 元野, 孙福兴, 赵明, 任浩, 朱广山, 化学学报, 2013, 71, 1598.); (b) Zhang, T. T.; Wang, H. T.; Ma, H. P.; Sun, F. X.; Cui, X. Q.; Zhu, G. S. Acta Chim. Sinica 2014, 72, 557. (张婷婷, 王海涛, 马和平, 孙 福兴, 崔小强, 朱广山, 化学学报, 2014, 72, 557.)

[3] Deng, W. Q.; Xu, X; Goddard, W. A. Phys. Rev. Lett. 2004, 92, 166103.

[4] Xu, X.; Goddard, W. A. Proc. Natl. Acad. Sci. 2004, 101, 2673.

[5] Frost, H.; Düren, T.; Snurr, R. Q. J. Phys. Chem. B 2006, 110, 9565.

[6] Frost, H.; Snurr, R. Q. J. Phys. Chem. C 2007, 111, 18794.

[7] Ryan, P.; Broadbelt, L. J.; Snurr, R. Q. Chem. Commun. 2008, 4132. 
[8] Han, S. S.; Choi, S. H.; Goddard, W. A. J. Phys. Chem. C 2010, 114, 12039.

[9] Bhatia, S. K.; Myers, A. L. Langmuir 2006, 22, 1688.

[10] Han, S. S.; Goddard, W. A. J. Am. Chem. Soc. 2007, 129, 8422.

[11] Sun, Q.; Jena. P.; Wang. Q.; Marquez, M. J. Am. Chem. Soc. 2006, 128,9741 .

[12] Wu, M. M.; Wang, Q.; Sun, Q.; Jena, P.; Kawazoe, Y. J. Chem. Phys. 2010, 133, 154706

[13] Li, A.; Lu, R. F., Wang, Y.; Wang, X.; Han, K. L.; Deng, W. Q. Angew. Chem. Int. Ed. 2010, 49, 3330.

[14] Yildirim, T.; Ciraci, S. Phys. Rev. Lett. 2005, 94, 175501.

[15] Konstas, K.; Taylor, J. W.; Thornton, A. W.; Doherty, C. M.; Lim, W. X.; Bastow, T. J.; Kennedy, D. F.; Wood, C. D.; Cox, B. J.; Hill, J. M.; Hill, A. J.; Hill, M. R. Angew. Chem. Int. Ed. 2012, 124, 6743.

[16] Klontzas, E.; Mavrandonakis, A.; Tylianakis, E.; Froudakis, G. E. Nano Lett. 2008, 8, 1572.

[17] Getman, R. B.; Miller, J. H.; Wang, K. J.; Snurr, R. Q. Phys. Chem. C 2011, 115, 2066.

[18] Zhou, J.; Wang, Q.; Sun, Q.; Jena, P.; Chen, X. S. Proc. Natl. Acad. Sci. 2010, 107, 2801 .

[19] Zhang, L. L.; Hu, Z. Q.; Jiang, J. W. J. Am. Chem. Soc. 2013, 135, 3722 .

[20] Ma, S.; Sun, D.; Simmons, J. M.; Collier, C. D.; Yuan, D.; Zhou, H. C. J. Am. Chem. Soc. 2008, 130, 1012.

[21] El-Kaderi, H. M.; Hunt, J. R.; Mendoza-Cortes, J. L.; Cote, A. P.; Taylor, R. E.; O'Keeffe, M.; Yaghi, O. M. Science 2007, 316, 268.

[22] Yuan, D.; Lu, W.; Zhao, D.; Zhou, H. C. Adv. Mater. 2011, 23, 3723.

[23] Duren, T.; Sarkisov, L.; Yaghi, O. M.; Snurr, R. Q. Langmuir 2004, 20,2683.

[24] Babarao, R.; Jiang, J.; Sandler, S. I. Langmuir 2009, 25, 5239.
[25] Jhon, Y. H.; Cho, M.; Jeon, H. R.; Park, I.; Chang, R.; Rowsell, J. L. C.; Kim, J. J. Phys. Chem. C 2007, 111, 16618.

[26] Lan, J. H.; Cao, D. P.; Wang, W. C. Langmuir 2010, 26, 220.

[27] Kowalczyk, P.; Brualla, L.; Zywocinski, A.; Bhatia, S. K. J. Phys. Chem. C 2007, 111, 5250.

[28] Wu, H.; Zhou, W.; Yildirim, T. J. Am. Chem. Soc. 2009, 131, 4995.

[29] Wu, H.; Simmons, J. M.; Liu, Y.; Brown, C. M.; Wang, X. S.; Ma, S.; Peterson, V. K.; Southon, P. D.; Kepert, C. J.; Zhou, H. C.; Yildirim, T.; Zhou, W. Chem. Eur. J. 2010, 16, 5205.

[30] Mendoza-Cortes, J. L.; Han, S. S.; Furukawa, H.; Yaghi, O. M.; Goddard, W. A. J. Phys. Chem. A 2010, 114, 10824.

[31] Wilmer, C. E.; Leaf, M.; Lee, C. Y.; Farha, O. K.; Hauser, B. G.; Hupp, J. T.; Snurr, R. Q. Nature Chem. 2012, 4, 83.

[32] Llewellyn, P. L.; Maurin, G.; Devic, T.; Loera-Serna, S.; Rosenbach, N.; Serre, C.; Bourrelly, S.; Horcajada, P.; Filinchuk, Y.; Ferey, G. $J$ Am. Chem. Soc. 2008, 130, 12808.

[33] Jiang, J. W.; Sandler, S. I. J. Am. Chem. Soc. 2005, 127, 11989.

[34] Liu, B.; Smit, B. Langmuir 2009, 25, 5918.

[35] Babarao, R.; Jiang, J. W. J. Am. Chem. Soc. 2009, 131, 11417.

[36] Lin, L. C.; Berger, A. H.; Martin, R. L.; Kim, J.; Swisher, J. A.; Jariwala, K.; Rycroft, C. H.; Bhown, A. S.; Deem, M. W.; Haranczyk, M.; Smit, B. Nature Mater. 2012, 11, 633.

[37] Kim, J.; Abouelnasr, M.; Lin, L.-C.; Smit, B. J. Am. Chem. Soc. 2013, 135, 7545.

[38] Krishna, R.; van Baten, J. M. Phys. Chem. Chem. Phys. 2011, 13 10593.

[39] Haldoupis, E.; Nair, S.; Sholl, D. S. J. Am. Chem. Soc. 2010, 132, 7528.

[40] Xie, Y.; Wang, T. T.; Liu, X. H.; Zou, K.; Deng, W. Q. Nature Commun. 2013, 4, 1960. 